\title{
Da Teoria à Prática da Interdisciplinaridade: a Experiência do Pró-Saúde Unifor e Seus Nove Cursos de Graduação
}

\author{
From Theory to Interdisciplinary Practice: the \\ Experience with the Pró-Saúde Unifor \\ Program and Undergraduate Courses
}

\author{
Magda Moura de Almeida \\ Rui Porto Morais ${ }^{I}$ \\ Danilo Frota Guimarães \\ Maria de Fátima Antero Sousa Machado ${ }^{I}$ \\ Rita de Cassia Moura Diniz \\ Sharmênia de Araújo Soares Nuto
}

\section{PALAVRAS-CHAVE: \\ - Ensino. \\ - Atenção Primária à Saúde. \\ - Serviços de Integração \\ Docente-Assistencial.}

\footnotetext{
KEYWORDS:

- Teaching.

- Primary Health Care.

- Teaching Care Integration Services.
}

Recebido em: 01/04/2010

Aprovado em: 26/08/2010

\section{RESUMO}

A Universidade de Fortaleza e a Secretaria de Saúde do município se vincularam ao Programa Nacional de Reorientação da Formação Profissional em Saúde (Pró-Saúde II), fortalecendo a parceria para reorientar a formação profissional por meio dos cursos de graduação do Centro de Ciências da Saúde da universidade. O projeto prioriza o desenvolvimento de ações interdisciplinares de assistência e atenção à saúde, proporcionando reforço aos Sistemas Locais Saúde-Escola nas áreas de abrangência de Centros de Saúde da Família, sensibilizando gestores e profissionais do serviço sobre a atuação interdisciplinar e implantando equipes ampliadas de saúde, buscando a educação permanente dos docentes, discentes e profissionais do serviço. Este artigo relata as ações e estratégias desenvolvidas neste contexto e produz uma reflexão acerca deste processo, mediante relato das mudanças nas estruturas curriculares nos cursos envolvidos e das atividades desenvolvidas nas unidades de saúde. Discute, ainda, aspectos que merecem ser aprofundados na interação ensino-serviço.

\begin{abstract}
The University of Fortaleza and the Fortaleza Municipal Health Department joined the National Program for Reorientation of Professional Training in Health (Pró-Saúde II), strengthening the partnership to reorient professional training through undergraduate courses at the university's Center for Health Sciences. The project prioritizes the development of interdisciplinary healthcare activities, thus reinforcing the Academic Local Health Systems in the coverage areas of the Family Health Centers, raising the awareness of policy-makers and health services staff concerning interdisciplinary action and deploying expanded health teams, seeking continuing education for faculty, students, and health services staff. This article reports on the actions and strategies developed in this context and reflects on the process by reporting the changes in the various course curricula and the activities conducted in the health units. The article further discusses issues that merit more in-depth investigation in the interface between teaching and health services.
\end{abstract}

ABSTRACT 


\section{INTRODUÇÃO}

Historicamente, os profissionais da área da saúde tiveram como cenários de prática em seus cursos de graduação, notadamente, ambientes hospitalares e clínicas, em que predominava um modelo de atenção individualizado e especializado. Esses profissionais, portanto, estavam pouco preparados para atuar junto aos problemas de saúde da coletividade e, mais recentemente, no Sistema Único de Saúde (SUS).

Esse contexto, associado às políticas públicas de saúde e de educação que envolvem a formação dos profissionais de saúde - relatadas nas Diretrizes Curriculares Nacionais $(\mathrm{DCN})^{1-9}$, no Aprender SUS, no Curso de Ativação de Mudanças para a Formação de Profissionais da Saúde (Fiocruz e MS), nas diretrizes da Comissão Intersetorial de Recursos Humanos do Conselho Nacional de Saúde (CIRH/CNS) e no Fórum Nacional de Educação dos Profissionais da Saúde (FNEPAS) -, vem mostrando, nos últimos anos, a necessidade de implementar mudanças nos cursos de graduação em saúde a partir de ações como, por exemplo, a ampliação dos cenários de prática para toda a rede municipal, práticas interdisciplinares e adoção de metodologias ativas no processo ensino-aprendizagem, objetivando transformar o processo de trabalho e ampliar a cobertura dos serviços de maneira a obter efeitos imediatos na qualidade das futuras gerações de profissionais de saúde das diferentes áreas.

A Universidade de Fortaleza (Unifor), há mais de 30 anos, desenvolve atividades por meio de seus cursos de graduação no Bairro Edson Queiroz (Comunidade do Dendê), fortalecendo sua parceria com a Secretaria Municipal de Saúde (SMS) de Fortaleza (CE). Essa parceria visa implementar e fortalecer a construção coletiva do Sistema Municipal de Saúde-Escola (SMSE), como política estruturante de organização e de gestão do serviço. Envolve a integração de potenciais humanos e de equipamentos, bem como a utilização de todos os espaços de produção da atenção à saúde e de produção da educação existentes no território municipal.

A principal ideia norteadora da SMS é a potencialização efetiva da reorientação do modelo assistencial, propiciando mudanças qualitativas no processo de trabalho em saúde; alterações estas contextualizadas numa vivência educacional que tem como protagonistas: gestores, trabalhadores da saúde, instituições formadoras e as diferentes representações dos movimentos sociais e populares.

OSMSE propõe-se a compartilhar entre os serviços de saúde e as instituições formadoras a responsabilidade sanitária de seu território e a educação continuada de seus profissionais. $\mathrm{O}$ município de Fortaleza está dividido em seis regiões administrativas, denominadas Secretarias Executivas Regionais (SER), que desempenham o papel executivo das políticas setoriais, que, de forma articulada, definem suas prioridades, estabelecendo metas específicas para cada grupo populacional e prestando serviços articulados em uma rede de proteção social. Cada SER funciona com um Distrito de Saúde, Educação, Meio Ambiente, Finanças, Assistência Social e Infraestrutura ${ }^{10,11}$.

Assim, Fortaleza foi territorializada e as SER foram delimitadas como áreas de responsabilidade sanitária das instituições de ensino, especificamente no que se refere à Atenção Primária à Saúde. O critério de distribuição das SER por universidade considerou a inserção desta no espaço geográfico e sua área de abrangência.

Como resultado desta parceria, a Unifor se insere nesse Sistema participando diretamente das redes de cuidados aos diferentes ciclos de vida por meio de estágios em hospitais distritais, das atividades do Núcleo de Assistência Médica Integrada (Nami) com a oferta de ambulatórios e exames complementares especializados, e, especial e preponderantemente, da articulação ensino-serviço da rede de Atenção Primária, com atividades docente-assistenciais em 16 Centros de Saúde da Família.

Como integrantes do SMSE de Fortaleza, a Unifor e a SMS se submeteram ao Edital de Convocação nº 13/2007 (Segetes), Programa Nacional de Reorientação da Formação Profissional em Saúde (Pró-Saúde II), fortalecendo a parceria, bem como: promovendo a reorientação da formação profissional em saúde para os alunos dos cursos de graduação do Centro de Ciências da Saúde/Unifor; focando ações interdisciplinares de assistência e atenção à saúde; implementando os sistemas locais saúde-escola nas áreas de abrangência de Centros de Saúde da Família da SER VI; promovendo a integração entre ensino e serviço; sensibilizando gestores e profissionais do serviço sobre a atuação interdisciplinar; e implantando equipes ampliadas de saúde - buscando, enfim, promover a educação permanente dos docentes, discentes e profissionais do serviço.

Este artigo relata as ações e estratégias que estão sendo desenvolvidas pelo Pró-Saúde/Unifor e produz uma reflexão acerca deste processo no contexto ensino-serviço.

\section{REORIENTAÇÃO CURRICULAR}

O Centro de Ciências da Saúde (CCS) possui nove cursos de graduação: Ciências da Nutrição (482 alunos); Enfermagem (1.535 alunos); Educação Física (396 alunos); Farmácia (549 alunos); Fisioterapia (986 alunos); Fonoaudiologia (197 alunos); Medicina (477 alunos); Odontologia (625 alunos); Terapia Ocupacional (160 alunos). Conta com 436 professores, dos quais $377(77,29 \%)$ são mestres e doutores. O corpo discente perfaz o total de 5.407 alunos regularmente matriculados. 
Os cursos de graduação se encontram em processo de mudança curricular, a fim de integrar os conteúdos disciplinares, inovar nas metodologias pedagógicas e se adequar ao perfil profissiográfico recomendado pelas $\mathrm{DCN}^{1-9}$.

Os projetos pedagógicos oriundos dessas mudanças passarão a contemplar a diversificação dos cenários de prática, bem como a inserção precoce do estudante nos espaços de saúde e redes sociais de apoio. Entretanto, há algumas dificuldades na integração ensino-serviço, como a falta de infraestrutura das unidades de atenção básica. Estas não oferecem espaço físico adequado para albergar docentes, discentes e os profissionais do serviço, nem dispõem de alguns profissionais de saúde nas equipes, como fonoaudiológos, nutricionistas, terapeutas ocupacionais, farmacêuticos, fisioterapeutas e educadores físicos.

Conforme o que as DCN preconizam para os cursos da área da saúde, buscamos estruturar o processo de formação do estudante segundo uma concepção mais ampla de saúde, estabelecendo como horizonte desejável para a organização curricular a integração das várias áreas do saber, tendo em vista a aprendizagem significativa. Esta estratégia possibilita superar a organização disciplinar, articulando os objetivos de aprendizagem em torno de temáticas relevantes e estimulantes. As DCN apontam também a necessidade de um papel ativo dos estudantes no processo de ensino-aprendizagem, para que este se torne ativo e independente, superando a dicotomia entre teoria e prática, valorizando o trabalho articulado com os serviços de saúde e grupos populacionais, bem como a interprofissionalidade ${ }^{1-9}$.

Para a organização curricular dos cursos de graduação na saúde, as DCN assinalam a necessidade de formar profissionais generalistas, humanistas, críticos e reflexivos, e capazes, dentro de princípios éticos, de promover a saúde integral do ser humano e trabalhar em equipe ${ }^{1-9}$.

Em 2005, o curso de Odontologia da Unifor iniciou uma proposta de integração curricular em que as habilidades gerais e específicas previstas na formação do cirurgião-dentista são exercitadas nas disciplinas das áreas de Ciências Biológicas e da Saúde, Ciências Humanas e Sociais, e Ciências Odontológicas. A estrutura curricular deixa de ser centrada em especialidades odontológicas e se orienta por perfil de complexidade crescente, seja do paciente, na área clínica, seja do campo de atuação, na Saúde Coletiva. O Sistema Único de Saúde se torna um conteúdo vertical que perpassa todos os semestres ${ }^{12}$.

Sendo o curso mais recente do CCS/Unifor, a graduação em Medicina já se inicia com uma proposta curricular diferenciada em 2006. Ciente do seu papel social e de que a simples incorporação de conteúdos das disciplinas da Saúde Coletiva ou mesmo a organização de estágios em atividades práticas da área podem não implicar uma mudança significativa no perfil do egresso do curso médico, o curso de Medicina investiu na introdução de novas abordagens pedagógicas do processo de ensino-aprendizagem, como a associação entre a Aprendizagem Baseada em Problemas (ABP), a metodologia da problematização e a educação baseada na comunidade (community based education), para que o aluno pudesse reorganizar seu processo pedagógico a partir da reflexão sobre o significado deste em sua vida.

Assim, nasceu o curso de Medicina na Universidade de Fortaleza, distribuindo seu currículo entre três amplas estratégias Ações Integradas em Saúde (AIS), Laboratório de Habilidades (LH) e Grupos Tutoriais (GT). Elas são divididas, semestralmente, em cinco módulos, sendo três sequenciais e relativos aos GT, com duração de seis semanas cada, e dois longitudinais, referentes às AIS e ao LH, durando 18 semanas cada um.

O curso de Medicina, em processo de implantação, hoje ofertando o oitavo semestre, se situa, desde o início, como elemento propulsor na integração das ações de saúde, considerando que a inserção dos alunos na Atenção Primária começa no primeiro semestre do curso, seguindo até o internato. Todas as ações neste nível de atenção são planejadas no módulo/ disciplina intitulado Ações Integradas de Saúde (AIS), de tal forma que os alunos desenvolvam suas práticas em nível de complexidade crescente.

Durante os anos em que se priorizam os conhecimentos das ciências básicas, os módulos de AIS buscam demonstrar, mediante experiências clínicas, a complexidade sistêmica do processo de adoecimento, evitando o modelo reducionista de "saúde-doença", como ocorre na seleção viciosa dos problemas vivenciados somente no hospital terciário.

A estratégia leva em conta toda a matriz curricular, além do internato em saúde coletiva e mental, por oito módulos nos quais o estudante de Medicina participa, sob orientação presencial de docentes, de atividades em espaços sociais variados - associações de moradores, igrejas, ONGs, rádios comunitárias, empresas - e em serviços de saúde de Atenção Primária. Estas atividades são planejadas e pactuadas procurando manter a adequação ao nível de resolução, aos objetivos de aprendizagem dos estudantes e às necessidades da população atendida nos diversos aparelhos sociais utilizados. Os módulos acontecem de maneira longitudinal, acompanhando cada semestre do curso. Cada um deles tem um eixo temático principal e norteador, que procura se integrar aos conteúdos abordados nas outras estratégias do curso. 
A partir das experiências exitosas dos cursos de Odontologia e Medicina, os demais cursos do CCS, ao iniciarem seu processo de mudança curricular, apresentam como eixo estruturante da proposta a formação dos profissionais das áreas da saúde que atuam no magistério. Desta forma, cumpre seu papel de investir na profissionalização do professor, proporcionando ao docente embasamento no campo epistemológico da educação, conferindo-lhe suporte para o trabalho pautado nos princípios pedagógicos, normalmente não contemplados nos cursos de formação em saúde ${ }^{13}$.

A mudança é resultado de reflexões da comunidade acadêmica e da própria gestão da universidade, segundo o entendimento de que as instituições formadoras devem buscar, de maneira constante, a qualidade do ensino. Quando há uma percepção de que a instituição de ensino atingiu o nível de excelência e por isso não precisa de mudanças, este estado conclusivo, fechado e rígido deve ser questionado. A instituição deve estar em constante movimento e em processo de análise, para não incorrer em acomodação e inércia ${ }^{14}$.

Portanto, a Unifor investe na formação continuada do corpo docente por meio de capacitação e consultorias para a mudança do currículo. Cada curso, por meio de seu grupo gestor, promove uma ampla discussão com todo o corpo docente para propor o perfil e as competências necessários à formação dos alunos.

Buscando a integração dos cursos e o desenvolvimento de competências intrínsecas à formação dos profissionais da saúde, o CCS propõe a estruturação de um núcleo comum que será oferecido no primeiro ano de todos os cursos, exceto Medicina, implantado recentemente, mas já moldado com metodologias ativas de ensino-aprendizagem e em processo de reconhecimento pelo MEC.

Estas são algumas das estratégias capazes de garantir, na prática, a interdisciplinaridade, a interprofissionalidade e o fortalecimento da integração ensino-serviço, onde todos esses elementos integram um movimento que resultará na melhoria da qualidade do ensino para a formação de profissionais da saúde comprometidos com as políticas de saúde e com as pessoas.

A busca da interdisciplinaridade como forma de atuação é o nosso intento, por acreditarmos nela como um instrumento que pode contribuir para tornar a escola um lugar onde se produza, coletiva e criticamente, um novo saber. Isto pressupõe interação e integração, não somente a superposição de disciplinas científicas, de seus conceitos e diretrizes, de sua metodologia e procedimentos, de seus dados e da organização de ensino.

É neste sentido da interdisciplinaridade que pensamos formar esses profissionais, pois, como dispõe Morin ${ }^{15}$, a realidade nos faz deparar hoje com problemas cada vez mais polidisciplinares, transversais, multidimensionais, transnacionais, globais e planetários, em relação aos quais os saberes separados, fragmentados, compartimentados entre disciplinas são inadequados, porquanto a inteligência que apenas sabe separar atrofia as possibilidades de compreensão e reflexão.

Urge refletir sobre o papel pedagógico da escola, pois a escola primária, como mostra Morin ${ }^{15}$, nos ensina a isolar os objetos, a separar as disciplinas e a dissociar os problemas, em vez de reunir e integrar, nos obriga a reduzir o complexo ao simples, isto é, a separar o que está ligado. O ensino universitário necessita de um novo modelo de educação mais problematizador ou conscientizador e integrado, que, ao contrário da educação bancária, objetiva o desenvolvimento da consciência crítica e da liberdade como meio de superar as contradições da realidade e responder à essência de ser da consciência, isto é, a sua intencionalidade.

Nesse sentido, na busca de uma prática atuante, precisamos tornar realidade a relação professor-aluno de forma horizontal, e não imposta, pois os alunos participam do processo juntamente com o professor. Com essa experiência acreditamos construir estratégias de formação de recursos humanos para o SUS do nosso país.

Alguns resultados já podem ser evidenciados, como: a integração intercursos; a formação de novos parceiros, a exemplo dos cursos de Comunicação Social, Direito e Psicologia; a assistência integral às famílias e à comunidade, visto que o atendimento prestado não é apenas multiprofissional, mas também interdisciplinar; acompanhamento não somente da população nos domicílios ou de usuários de serviços de saúde, mas também o acompanhamento de instituições, como creches, escolas, lares para idosos - alicerçados no conhecimento do perfil socioeconômico, cultural e epidemiológico das comunidades onde atuamos.

Com o aluno inserido precocemente em atividades práticas relevantes para sua futura vida profissional, além do crescimento pedagógico proporcionado na área de Saúde Coletiva, essa metodologia de intervenção tenta inverter a lógica do modelo de atenção, agora centrado na prevenção de doenças e promoção da saúde, trabalhando com os reais problemas da comunidade.

\section{DESENVOLVIMENTO DAS ATIVIDADES}

Para ofertar respostas concretas às necessidades de saúde da população por meio da cooperação entre gestores e equipes de saúde da família, reorientação da formação dos alunos de graduação do Centro de Ciências da Saúde (CCS) e qualificação dos serviços de saúde, o projeto Pró-Saúde/Unifor tem por objetivo geral a promoção e a reorientação da formação profissional em saúde para os cursos do CCS da Unifor, integrando a Estratégia de Saúde da Família (ESF), com ênfase em ações interdisciplinares de assistência e atenção à saúde. 
O Projeto Pró-Saúde/Unifor concentrou seus recursos para a reforma de três Centros de Saúde da Família, com compra de material permanente e consumo (uma unidade de saúde por ano). Apesar disso, o projeto tem buscado organizar a inserção dos alunos de graduação em toda a SER VI e a articulação das atividades curriculares com as atividades desenvolvidas no Projeto PET-Saúde/Unifor.

O Quadro 1 relaciona os Centros de Saúde da Família, com os cursos de graduação que desenvolvem atividades de disciplinas práticas, teórico-práticas e estágios, e o número de turmas. O CSF Maria de Lourdes Ribeiro Jereissate foi alvo da reforma no Ano I - Pró-Saúde/Unifor. No semestre 2009.1, tinha somente cinco turmas práticas dos cursos de Enfermagem, Odontologia e Fonoaudiologia. No semestre 2009.2, após a ampliação e a adequação do espaço físico para práticas pedagógicas, foi possível incorporar as atividades dos cursos de Medicina, Educação Física, Fisioterapia, Farmácia, Terapia Ocupacional e Nutrição, além dos existentes, possibilitando a inclusão de 16 turmas.

\section{QUADRO 1}

Centro de Saúde da Família: cursos e número de turmas práticas, SER VI, Fortaleza-CE, 2009.2

\begin{tabular}{|c|c|c|}
\hline $\begin{array}{c}\text { Centro de } \\
\text { Saúde da Família }\end{array}$ & Cursos & $\begin{array}{l}\text { No de } \\
\text { turmas }\end{array}$ \\
\hline Anísio Teixeira & Medicina & 2 \\
\hline César Cals & $\begin{array}{l}\text { Fisioterapia, Farmácia, } \\
\text { Enfermagem }\end{array}$ & 6 \\
\hline Edmar Fujita & $\begin{array}{l}\text { Enfermagem, Farmácia, } \\
\text { Odontologia }\end{array}$ & 6 \\
\hline Fco. Melo Jaborandi & $\begin{array}{l}\text { Odontologia, Nutrição, } \\
\text { Enfermagem }\end{array}$ & 4 \\
\hline Galba Araújo & Enfermagem & 1 \\
\hline Hélio Goes & Enfermagem, Odontologia & 4 \\
\hline Janival de Almeida & Enfermagem, Odontologia & 6 \\
\hline João Hipólito & Enfermagem & 1 \\
\hline José Barros & Enfermagem & 1 \\
\hline $\begin{array}{l}\text { Maria de Lourdes } \\
\text { Ribeiro Jereissate }\end{array}$ & $\begin{array}{l}\text { Enfermagem, Fisioterapia, } \\
\text { Farmácia, Odontologia, } \\
\text { Fonoaudiologia, Terapia } \\
\text { Ocupacional, Nutrição, Medicina }\end{array}$ & 16 \\
\hline Monteiro de Morais & Enfermagem, Medicina & 3 \\
\hline Messejana & Odontologia, Farmácia & 2 \\
\hline Pedro Sampaio & Enfermagem & 1 \\
\hline Terezinha Parente & $\begin{array}{l}\text { Odontologia,Enfermagem, } \\
\text { Fisioterapia, Medicina, } \\
\text { Fonoaudiologia, Terapia } \\
\text { Ocupacional, Nutrição }\end{array}$ & 11 \\
\hline Waldo Pessoa & $\begin{array}{l}\text { Enfermagem, Medicina, } \\
\text { Odontologia, Fisioterapia }\end{array}$ & 9 \\
\hline Matos Dourado & $\begin{array}{l}\text { Enfermagem, Fisioterapia, } \\
\text { Odontologia, Nutrição, Medicina }\end{array}$ & 22 \\
\hline
\end{tabular}

Nos 16 Centros de Saúde da Família da SER VI envolvidos no acompanhamento dos alunos dos nove cursos do CCS da Unifor, 96 profissionais do Sistema Municipal Saúde Escola participam do desenvolvimento das atividades docente-assistenciais. Destes profissionais, 18 são preceptores do PET-Saúde, e todos, mediante contrapartida da universidade, receberam acesso à Biblioteca Central e participam de atividades de educação permanente, incluídas na programação do Pró-Saúde/Unifor.

Além do tensionamento da mudança curricular e do fortalecimento da inserção cada vez mais precoce dos alunos dos cursos do CCS na Atenção Básica, o Projeto Pró-Saúde/Unifor tem contribuído para:

a) Estabelecer o vínculo serviço-ensino-pesquisa na Atenção Básica:

- Padronização de fluxo de projetos de pesquisa;

- Produção de CDs com os resultados das pesquisas desenvolvidas na Atenção Primária à Saúde;

- Disponibilização dos resumos das pesquisas realizadas na Atenção Primária à Saúde no site www.crescer.med.br/ prosaude.

b) Fortalecer a reorientação do modelo de atenção à saúde nos Centros de Saúde da Família (CSF) da SER VI:

- Confecção de cartilhas educativas para orientar as atividades desenvolvidas pelos diversos cursos;

- Desenvolvimento de fluxograma para as atividades docentes-assistenciais;

- Desenvolvimento de proposta de prontuário interdisciplinar.

c) Apoiar a implantação e sensibilizar gestores e profissionais do serviço sobre a atuação interdisciplinar, a partir das equipes ampliadas de saúde:

- Incentivar a participação dos docentes e discentes nas rodas de gestão das unidades de saúde, esclarecendo a atuação de cada profissão.

De acordo com a capacidade de cada CSF, a universidade negocia com a SER VI as unidades que podem receber as turmas das disciplinas práticas, teórico-práticas e estágios. Cada disciplina na Atenção Básica desenvolve atividades diagnósticas individuais e coletivas, de aprimoramento da assistência e atenção em saúde nas diversas áreas e de desenvolvimento de grupos educativos, buscando a promoção de saúde e visando fortalecer a interdisciplinaridade, na medida do possível. Estas atividades são consolidadas semestralmente, por unidade de saúde, por meio dos Relatórios de Consolidação de Atividades, em que constam as ações desenvolvidas pelos docentes e discentes da Unifor, e estão disponibilizadas no site www. crescer.med.br/prosaude. 
Desde que a Unifor foi contemplada no Edital Pró-Saúde II, as disciplinas e estágios que desenvolvem suas ações na Atenção Primária, junto com a coordenação do Pró-Saúde/ Unifor e o setor de Supervisão de Estágio do CCS, têm buscado fortalecer a relação ensino-serviço por intermédio da maior participação dos professores no planejamento das unidades de saúde (rodas), do compartilhamento das atividades desenvolvidas (consolidado de atividades semestrais), de planejamentos coletivos (Encontros de Saúde Coletiva) e das exposições de experiências exitosas (Mostras). Assim, a perspectiva de maior inserção da formação da graduação em saúde no SUS possibilita uma mudança na prática pedagógica tradicional, centrada em ambientes hospitalares e clínicos.

\section{A experiência de nove cursos com a educação baseada na comunidade no Nordeste brasileiro}

A mudança do perfil epidemiológico da população, com grande predomínio de doenças crônico-degenerativas, exigiu o reordenamento das ações e estratégias na saúde, com sérias implicações na formação dos profissionais. Cada vez mais, o trabalho multiprofissional e os conhecimentos interdisciplinares se tornam necessários para enfrentar as complexas necessidades de saúde das comunidades. Os conhecimentos e práticas da saúde coletiva, bem como o entendimento e a participação na construção das políticas públicas e na organização dos serviços de saúde se tornam competências imprescindíveis ao desempenho dos profissionais da saúde. Assim, o Pró-Saúde/Unifor também aproxima a formação de graduação no País das necessidades da atenção básica, que se traduzem no Brasil pela Estratégia de Saúde da Família (ESF).

Este enfoque sobre problemas prioritários fornece ao aluno o poder de análise dos determinantes e componentes das situações de saúde. No entanto, nesse confronto com a realidade, a qualidade da informação repassada ao estudante deve ser da maior credibilidade possível. Esta confiabilidade se constrói por meio da alta resolutividade do serviço e da competência técnico-humanistica dos profissionais, agregadas à satisfação do usuário. O trabalho em equipes multi e interdisciplinares, além de treinar o estudante em habilidades como gerenciamento, coordenação, e liderança e em atitudes como cooperação, também agrega valor ao serviço.

Nessa perspectiva, a Unifor implantou o Pró-Saúde, com a participação dos nove cursos do CCS, envolvendo em torno de 450 alunos de graduação e 125 professores de disciplinas / módulos práticos, alicerçados na educação baseada na comunidade (community based education), que envolve a utilização da comunidade durante toda a experiência educativa.
A principal limitação encontrada para uma iniciativa deste porte se encontrava na infraestrutura dos espaços de prática, voltados para atividades eminentemente assistenciais, individuais e uniprofissionais. Com ajuda da verba governamental, a Unifor investirá em três cenários de prática nas proximidades da universidade: o Centro de Saúde da Família Maria de Lourdes Jereissate (CSF-ML), o CSF Francisco Melo Jaborandi e o CSF Terezinha Parente.

O CSF-ML foi ampliado e reformado para oferecer condições favoráveis ao ensino adequado e ao desenvolvimento de projetos que visam à melhoria das condições de saúde da população de acordo com a demanda do serviço, nunca se esquecendo da prática de prevenção, promoção e reabilitação em saúde.

Cada curso de graduação envolvido possui um monitor bolsista discente que tem como tutor um docente de seu curso. Este professor ministra disciplinas/módulos teórico-práticos na referida unidade e tem a função de facilitar a aprendizagem ativa do aluno, orientando-o durante os trabalhos interdisciplinares.

Durante esse tempo de experiência, foi constatada uma satisfatória adequação das práticas de ensino às atividades assistenciais desenvolvidas nessa unidade, com a ampliação da oferta de serviços de Atenção Primária. Como frutos desta bem-sucedida relação de ensino-serviço-comunidade podemos citar:

- Interconsulta médico-nutricional para acompanhamento de pacientes com doenças cardiometabólicas e crianças portadoras de anemia e déficit de crescimento ou desenvolvimento;

- Interconsulta médico-odontológica, com estabelecimento de Protocolos de Atenção à Saúde Bucal para Hipertensos e Diabéticos;

- Pós-consulta farmacêutica, inserção da Atenção Farmacêutica nos cuidados dos pacientes crônicos;

- Processo de Educação Permanente para as auxiliares de enfermagem, com oficinas direcionadas para aferição das medidas antropométricas, aferição de pressão arterial e utilização de equipamentos de proteção individual;

- Aumento da cobertura e melhoria da resolutividade dos pacientes em cuidados domiciliares em todas as áreas da saúde;

- Procedimentos ambulatoriais, instituindo a resolução na própria unidade de problemas dermatológicos e cirúrgicos de baixa complexidade, como cistos sebáceos, drenagem de abscessos, pequenos lipomas, suturas, etc.;

- Cursos de gestantes interdisciplinares;

- Estruturação do grupo de idosos, hipertensos e diabéticos da comunidade, com apoio das áreas de Educação Física, Nutrição, Fisioterapia e médica;

- Estruturação dos grupos com necessidades específicas nas áreas de Fonoaudiologia e Terapia Ocupacional 


\section{AVANÇOS E DESAFIOS}

A busca do aumento da inserção da formação da graduação em saúde no SUS tem resultado em avanços e desafios aos diversos cursos do CCS/Unifor:

- Enfermagem e Odontologia: fortaleceram-se na atuação em Saúde Coletiva, mas precisam de espaços clínicos e maior infraestrutura para formação clínica na Atenção Primária à Saúde;

- Medicina: desenvolve ampla atuação na Atenção Primária à Saúde em espaços clínicos, mas a participação do docente médico nos espaços de planejamento e atuação interdisciplinar ainda é muito restrita. Existe também um tensionamento, geralmente negativo, entre o corpo docente/discente e o profissional médico do serviço, que se sente ameaçado em seu campo de trabalho pelas intervenções reflexivas trazidas pelos estudantes e pelo corpo docente-assistencial sobre estruturas e práticas organizacionais nas quais estes indivíduos se encontram imersos e engessados. Essa postura tende a dificultar a efetiva mudança na organização dos serviços, não apenas em termos do processo de ensino-aprendizagem na APS, mas também do aprimoramento da qualidade e resolutividade do atendimento aos usuários;

- Fisioterapia, Farmácia, Fonoaudiologia, Terapia Ocupacional, Educação Física e Nutrição: precisam sedimentar o papel de cada profissão na Atenção Primária à Saúde. Todos estes cursos já avançaram bastante, pois os alunos desenvolvem disciplinas e/ou estágios nos Centros de Saúde da Família; entretanto, o reconhecimento e a atuação profissional neste nível de atenção ainda não estão bem articulados com a rede de serviços de saúde do município e seus profissionais;

- O maior desafio é o fortalecimento da atuação interdisciplinar, incluindo maior parceria com os Núcleos de Apoio à Saúde da Família (Nasf).

Acreditamos que, mesmo com estes desafios, estamos caminhando para tornar possível a integração de todos os processos de formação da rede (educação permanente, graduações, especializações e residências). O primeiro passo já está dado. Parafraseando o grande poeta da modernidade Chico Science: "Um passo à frente e você não está mais no mesmo lugar".

\section{REFERÊNCIAS}

1. Brasil. Ministério da Educação. Diretrizes nacionais do curso de graduação em Enfermagem. Resolução CNE/ CES de 7 de novembro de 2001. DOU de 09 de novembro de 2001, seção 1, p. 37. Brasília, 2001.

2. Brasil. Ministério da Educação. Diretrizes nacionais do curso de graduação em Medicina. Resolução CNE/CES de 7 de novembro de 2001. DOU de 09 de novembro de 2001, seção 1, p. 38. Brasília, 2001.
3. Brasil. Ministério da Educação. Diretrizes nacionais do curso de graduação em Nutrição. Resolução CNE/CES de 7 de novembro de 2001. DOU de 09 de novembro de 2001, seção 1, p. 39. Brasília, 2001.

4. Brasil. Ministério da Educação. Diretrizes nacionais do curso de graduação em Odontologia. Resolução CNE/CES de 19 de fevereiro de 2002. DOU de 4 de março de 2002, seção 1, p. 10. Brasília, 2002.

5. Brasil. Ministério da Educação. Diretrizes nacionais do curso de graduação em Farmácia. Resolução CNE/CES de 19 de fevereiro de 2002. DOU de 4 de março de 2002, seção 1, p. 9. Brasília, 2002.

6. Brasil. Ministério da Educação. Diretrizes nacionais dos cursos de graduação em Fisioterapia. Resolução CNE/ CES de 19 de fevereiro de 2002. DOU de 04 de março de 2002, seção 1, p. 11. Brasília, 2002.

7. Brasil. Ministério da Educação. Diretrizes nacionais dos cursos de graduação em Fonoaudiologia. Resolução CNE/ CES de 19 de fevereiro de 2002. DOU de 04 de março de 2002, seção 1, p. 12. Brasília, 2002.

8. Brasil. Ministério da Educação. Diretrizes nacionais dos cursos de graduação em Terapia Ocupacional. Resolução CNE/CES de 19 de fevereiro de 2002. DOU de 04 de março de 2002, seção 1, p. 12. Brasília, 2002.

9. Brasil. Ministério da Educação. Diretrizes nacionais do curso de graduação em Educação Física, em nível superior de graduação plena. Resolução CNE/CES de 14 de junho de 2007. DOU de 24 de setembro de 2007, seção 1, p. 10. Brasília, 2007.

10. Fortaleza. Secretaria Municipal de Saúde. Relatório de gestão do ano de 2005 da Secretaria Municipal de Saúde de Fortaleza: saúde, qualidade de vida e a ética do cuidado. Fortaleza: SMS; 2006. Mimeo.

11. Fortaleza. Secretaria Municipal de Saúde. Relatório de gestão do ano de 2006 da Secretaria Municipal de Saúde de Fortaleza: saúde, qualidade de vida e a ética do cuidado. Fortaleza, CE: SMS; 2007. Mimeo.

12. Noro LRA, Nuto SAS, Moreira TP, Moura KS, Novais PMR. Projeto pedagógico: a construção coletiva. In: Noro LRA, org. Curso de Odontologia da UNIFOR: 10 anos ensinando e aprendendo. Fortaleza: Universidade de Fortaleza; 2005. p. 19-39.

13. Arroyo MG. Ofício de mestre: imagens e auto-imagens. Petrópolis: Vozes; 2000.

14. Tardif M. Saberes profissionais dos professores e conhecimentos universitários. Rev Bras Educ. 2000.

15. Morin E. A cabeça bem feita: repensar a reforma e reformar o pensamento. 5 ed. Rio de Janeiro: Bertrand; 2001. 


\section{CONTRIBUIÇÃO DOS AUTORES}

Todos os autores contribuirão na concepção e desenho do artigo,da análise e interpretação dos dados assim como da redação deste texto.

\section{CONFLITO DE INTERESSES}

Declarou não haver.

\section{ENDEREÇO PARA CORRESPONDÊNCIA}

Magda Moura de Almeida

Rua Ônix, 24

Papicu - Fortaleza

CEP. 60175-730 CE

E-mail: magda@unifor.br 